\title{
Champs tectoniques
}

\author{
Tectonic fields
}

\author{
P. SIRIEYS \\ Laboratoire de Géologie Alpine - URA 69 du CNRS \\ Institut Dolomieu*
}

Rev. Franç. Géotech. n 56, pp. 15-22 (juillet 1991)

\section{Résumé}

La tectonique quantitative dont l'objet est la reconstitution des champs cinématiques des massifs rocheux et, éventuellement, des champs de contraintes, s'appuie sur la géométrie des structures actuelles, qui résulte de déplacements et déformations continus et/ou discontinus (structures plissées et/ou faillées). L'analyse cinématique permet à l'aide de marqueurs (par exemple objets et fossiles déformés, orientation et rejets de failles) la détermination des tenseurs de déformation finie $D$ et de déformation progressive $D(t)$ lou " chemin de la déformation $n$ ), de la déformation homogénéisée (ou régionale) résultant de discontinuités de déplacements et des superpositions séquentielles des déformations tectoniques. Ces champs cinématiques sont enfin reliés aux champs de paléocontraintes.

Ces études apportent des informations (notamment sur l'anisotropie des roches, les surfaces éventuelles de rupture, les champs de contraintes naturels) essentielles dans les études géomécaniques.

\section{Abstract}

The purpose of the quantitative tectonic is the reconstruction of kinematic fields of rocks masses and, if possible, of the stressfields. It is founded upon the geometry of actual structures, which results in continuous and/or discontinuous displacements and strains (folded and/or faulted structures). The kinematic analysis enables, with the help of markers (for example strained objects and fossils, orientation and slip or faults), the determination of (i) the finite strain tensor $D$, (ii) the progressive strain D (t) (or « strain path ") (iii) the homogeneous strain resulting in discontinuous displacements, (iv) the superimposed sequences of tectonic strains. These kinematic fields are connected to paleostress fields.

These studies involve information essential to the geomechanical studies, especially for the anisotropy of rocks, the possible surfaces of break, and the natural stressfields. 


\section{INTRODUCTION}

L'observation sur affleurement et au cours de la réalisation d'ouvrages montre fréquemment des roches déformées et des structures qui jouent un rôle majeur dans les études et projets.

La déformation naturelle des roches peut être déterminée à partir de mesures in situ sur des marqueurs de types variés. Elle peut également être reproduite en laboratoire : essais sous haute pression et température permettant de réaliser des déformations importantes homogènes ou hétérogènes (kink-bands par exemple). Toutefois, pour représenter la déformation tectonique l'expérimentation présente des limites du fait des difficultés dans la réalisation de déformations rotationnelles et surtout d'essais aux vitesses de déformation naturelle (inférieures à $10^{-12} \mathrm{sec}^{-1}$ ). Les tendances actuelles sont les essais sur modèles analogiques, l'interprétation des déformations naturelles (qui fournit des informations sur la rhéologie des roches, notamment dans des conditions inaccessibles à l'expérimentation) et la modélisation numérique.

\section{GÉOMÉTRIE DES STRUCTURES}

L'étude de la géométrie de l'état actuel, l'état déformé (outre son importance en géomécanique et géotechnique) est à la base des études de tectonique quantitative, qui visent à reconstituer les champs cinématiques (de déplacement et déformation). Les structures actuelles dépendent de l'hétérogénéité de la déformation naturelle qui se manifeste sous deux formes: continue et/ou discontinue.

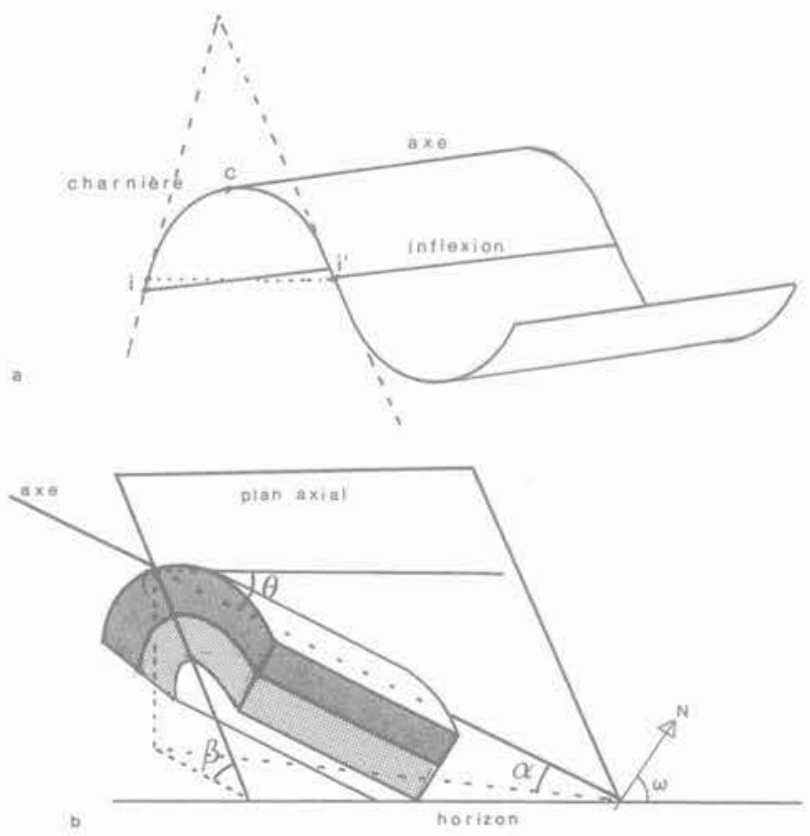

Fig. 1. - Plissement.

a. Charnière et flancs $\left(i, i^{\prime}=\right.$ points d'inflexion); b. Axe et plan axial; c. Plis non cylindriques $(S A=$ surface axiale $) ; d$. Polarité $\left(d_{1}=\right.$ anticlinal, $d_{2}=$ pli couché, $d_{3}=$ synforme anticlinale 1 Fig. 1 . - Folding

a. Hinge and limbs $\left(i, i^{\prime}=\right.$ inflexion points); b. Axis and axial plane ; c. Non cylindrical folds $(S A=$ Axial surface $): d$. Polarity $\left(d_{2}=\right.$ anticline $d_{2}=$ overturned fold,$d_{3}=$ anticlined synform).
1.1. Structures plissées (fig. 1)

Un cas fréquent d'hétérogénéité de la déformation continue est le plissement, qui résulte généralement d'une instabilité de la contraction finie d'un milieu initialement anisotrope (flambement), parfois d'une hétérogénéité du champ de déplacements (plis d'écoulement).

Une surface plissée (en général un joint de stratification, parfois une schistosité antérieure) est caractérisée, en section, par l'analyse de la courbure (inverse du rayon de courbure) qui atteint sa valeur maximale dans la zone de charnière, une valeur nulle au point d'inflexion dans le flanc du pli (fig. 1a).

Un plissement cylindrique est défini par sa surface axiale, souvent assimilable à un plan : le plan axial, qui regroupe les génératrices charnières, et son axe, génératrice lieu d'une charnière (fig. 1b).

Un premier élément caractéristique d'un plissement cylindrique à surface axiale plane est son orientation spatiale, dans les axes géographiques (fig. 1b). Elle fait intervenir trois angles : la direction $\omega$ du plan axial (par rapport au N), le pendage $\beta$ du plan axial, le pendage $\alpha$ de l'axe de pli. Le plongement $\theta$ (en anglais pitch) de l'axe de pli dans le plan axial est fréquemment utilisé. Il est relié à $\alpha$ et $\beta$ par la relation : $\sin \alpha=\sin \beta \cdot \sin \theta$ (le cas $\beta=\pi / 2$ et $\alpha=$ 0 caractérise un pli droit à axe horizontal, le cas $\beta$ $=\alpha=\pi / 2$ relatif à un plissement à plan axial et axes verticaux est également observé. Dans les études géomécaniques et géotechniques, la confrontation entre ces angles et ceux de l'orientation de l'axe d'un tunnel ou d'une tranchée est déjà d'une grande importance.
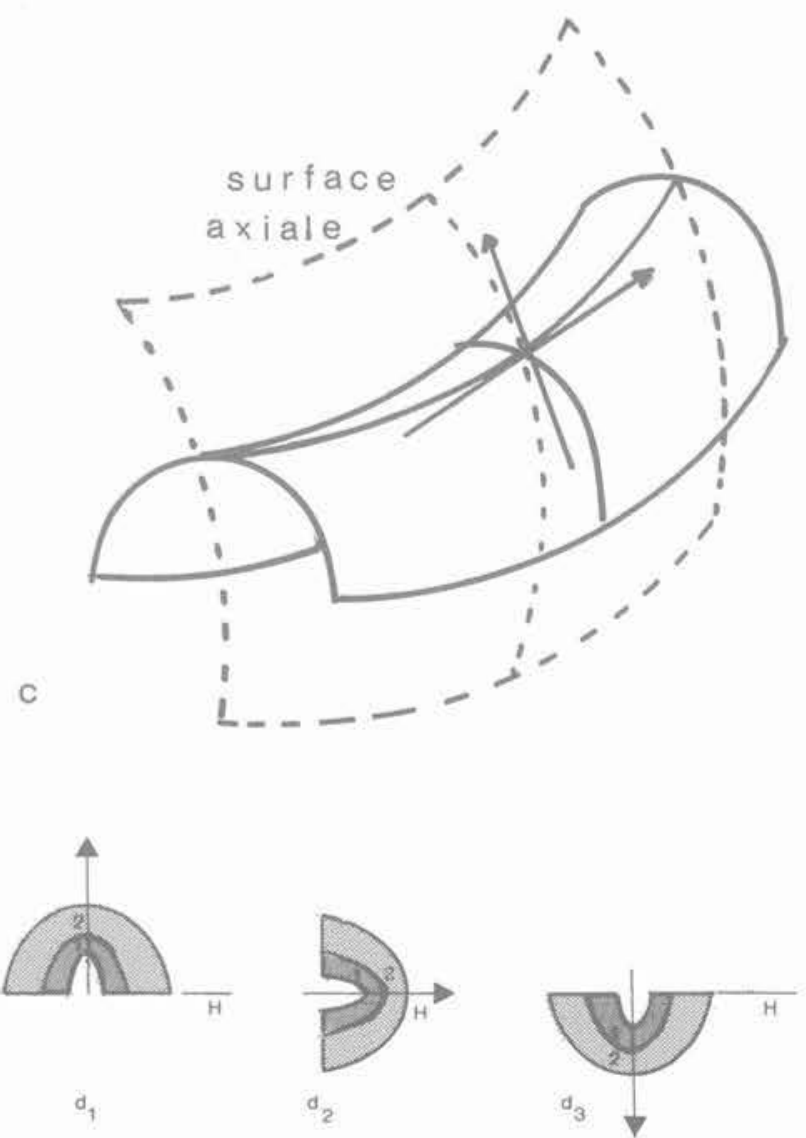
Un cas plus général concerne des surfaces plissées non réglées, ces plis sont alors définis par leur surface axiale, la ligne axiale, par exemple des plis replissés. Les angles précédents sont alors valables localement (plan tangent axial) (fig. 1c).

Un second élément d'analyse géométrique d'une surface stratifiée plissée est la polarité des couches (déduite des âges des dépôts et du granoclassement). Par exemple, pour un plissement à axe horizontal $(\alpha=0)$, le cas $\beta=-\pi / 2$ caractérise une antiforme anticlinale (fig. 1d).

Enfin, une autre caractéristique des multicouches plissées est, en section, la variation d'épaisseur des couches, qui conduit à une classification des plis selon leur épaisseur (RAMSAY, 1967), comportant notamment les plis isopaques (ou parallèles) et les plis semblables.

L'hétérogénéité de la déformation peut se manifester par une localisation: cas des kink-bands, type de plis particuliers, ou de bandes de glissement.

\subsection{Structures fracturées (fig. 2)}

Les déplacements peuvent présenter une discontinuité sur des surfaces discrètes, souvent planes, regroupées alors en familles de plans parallèles. La discontinuité affecte soit la composante du déplacement normale au plan, elle caractérise alors une famille de diaclases (rupture en mode I), soit la composante tangentielle, elle caractérise alors une famille de failles (rupture en mode II), ce second cas pouvant représenter une bande de cisaillement d'épaisseur nulle. La dis-

a
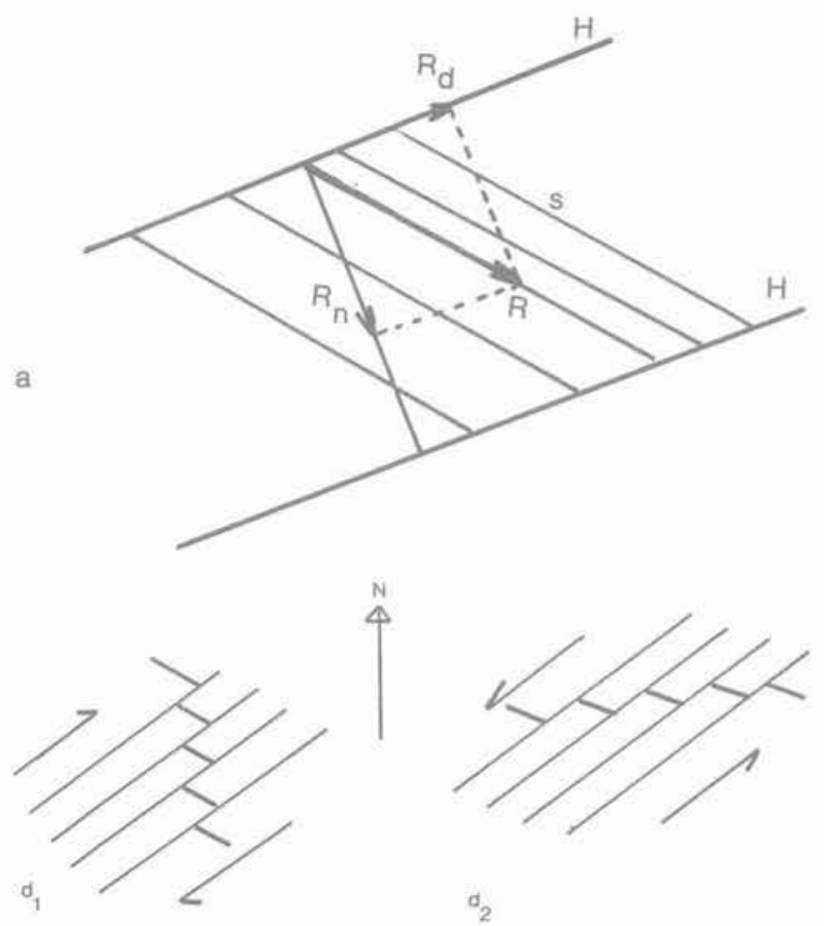

continuité est caractérisée par le rejet, souvent repéré, dans le plan de faille, par ses deux composantes: extensive (ou contractive) $R_{n}$ et décrochante $R_{d}$ (fig. $2 \mathrm{a}$ ). $\mathrm{R}_{\mathrm{d}}$ nul caractérise une famille de failles normales (fig. $2 b$ ) ou inverses (fig. $2 c$ ). $R_{n}$ nul caractérise des décrochements purs, dextres (fig. $d_{1}$ ) ou sénestres (fig, $\mathrm{d}_{2}$ ).

\subsection{Structures mixtes}

Les deux mécanismes de déplacements continus et discontinus peuvent coexister, qu'ils se soient effectués simultanément ou successivement. Exemple : le plissement avec glissement banc sur banc (fig. 3), les zones de décrochements utilisées par des cluses.

\section{LA DÉFORMATION}

Les études de tectonique quantitative, déduites de l'étude géométrique des structures actuelles, consistent à déterminer la déformation finie, en général rotationnelle, entre état initial reconstruit et état actuel et son chemin.

\subsection{Déformation finie}

Dans la configuration initiale, la déformation finie est définie à partir des gradients des coordonnées par :

$$
\mathrm{T}=\frac{\vartheta \xi_{1}}{\vartheta \mathrm{x}_{\mathrm{k}}}
$$

$\mathrm{x}, \xi$ sont les coordonnées dans l'état initial, final.

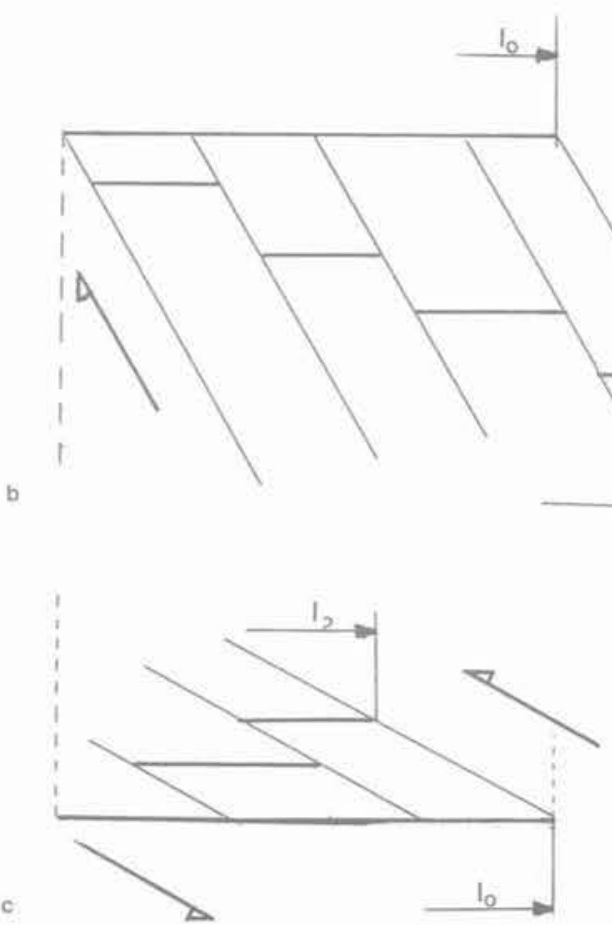

Fig. 2. - Déplacements discontinus.

a. Rejet: composante normale $R_{n}$ et décrochante $R_{d} ; b$. Failles normales (tectonique extensive) : c. Failles inverses (tectonique contractive); $d$. Décrochements $\left(d_{1}=\right.$ dextre, $d_{2}=$ sénestre).

Fig. 2. - Discontinuous displacements.

a. Net slip: $R_{n}$ : dip slip component; $R_{d}$ : strike slip component.

b. Normal faults (extension tectonic); $c$. Peverse faults (shortening tectonic); $d$. Strike slip fault $\left.(d)=d e x t r a l, d d_{2}=s i n e s t r a l\right)$. 


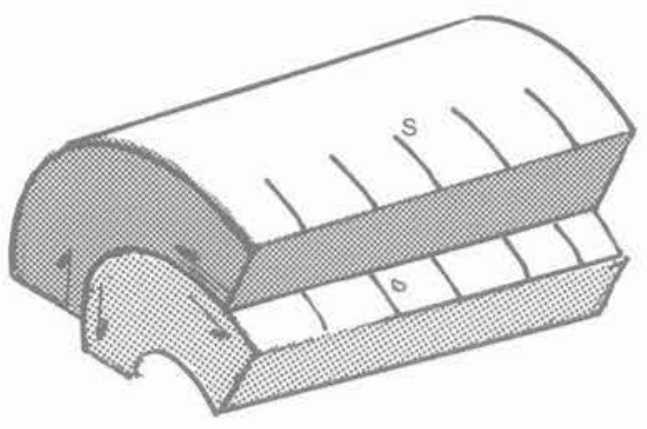

Fig. 3. - Plissement avec glissement banc sur banc $(s=$ strie $)$.

Fig. 3. - Folding with intrabeding sliding ( $s=$ striae).

Par décomposition polaire :

$$
T=R D=\bar{D} R
$$

sont définies la rotation rigide (externe) $\mathrm{R}$, la déformation avant rotation (D), après rotation $(\bar{D})$.

La déformation pure $D$ (ou $\bar{D}$ ) étant supposée isovolume est donc d'un des deux types: schisteuse (deux extensions principales et une contraction) ou fibreuse (une extension et deux contractions). Le cas intermédiaire (une déformation principale nulle) caractérise un état de déformation plane. Elle est représentée (fig. 4) dans un diagramme ternaire. La géométrie de la déformation bidimensionnelle est imagée par son cercle de Mohr ou de Weyrauch.

Dans la configuration actuelle, la déformation est caractérisée par la transformation inverse $\mathrm{T}^{-1}$. Dans ce cas, les études sont dites de "retrotectonique ».

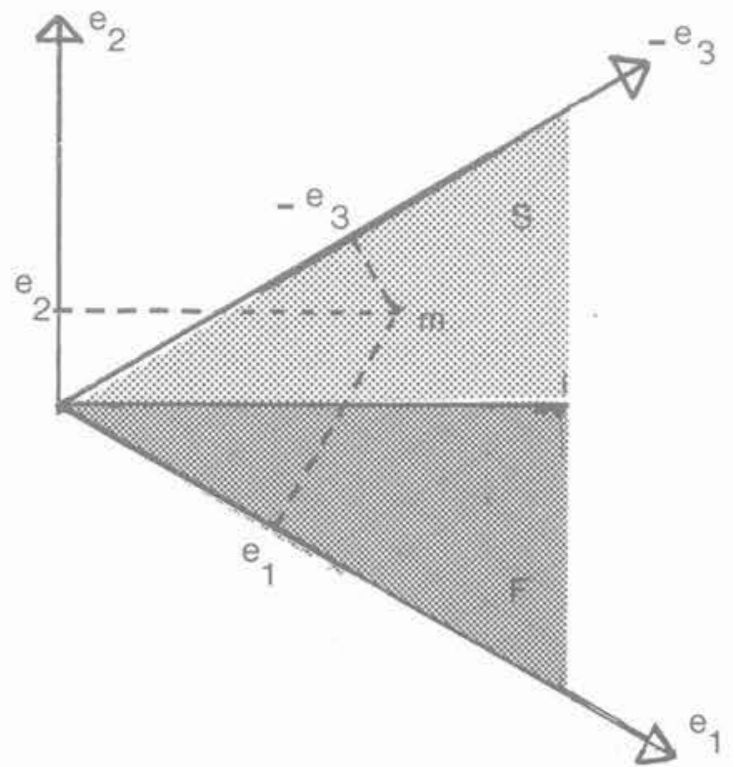

Fig. 4. - Déformation finie. Diagramme ternaire $e_{1}, e_{2}, e_{3}=$ déformations principales logarithmiques, $S=$ schistosité $; F=$ fibrosité).

Fig. 4. - Finite strain. Ternary diagram

$\left(e_{1}, e_{2}, e_{3}=\right.$ principal logarithmic strains, $S=$ cleavage, $F=$ lineation .
Lorsque la déformation résulte de déplacements discontinus, elle est déterminée à partir des gradients des rejets et appelée déformation homogénéisée (SIRIEYS, 1984), elle caractérise, en direction et en intensité, la déformation continue équivalente qui s'effectuerait sans localisation. On notera que la déformation homogénéisée peut être hétérogène.

La déformation se définit donc à différentes échelles et il convient de distinguer entre déformation homogénéisée (ou régionale, ou globale) et la déformation lcoale (ou interne) qui peut éventuellement être nulle (schémas de blocs).

\subsection{La déformation progressive}

L'étude de la déformation finie, entre état initial et état final, est insuffisante pour la décrire en totalité puisque entre les deux configurations, une infinité de chemins conduisent à une même forme (mais généralement avec des structures différentes). Par exemple (fig. 5a) : à partir d'un cercle dans l'état initial, l'ellipse finale peut être obtenue par déformation pure, glissement simple (rotationnel), déformation pure superposée à une rotation (chemin biphasé). Sa connaissance totale nécessite l'étude de sa chronologie, c'est-à-dire de la déformation progressive (GOGUEL, 1979), à l'aide de la vitesse de déformation ou de transformation $\mathfrak{I}$ définie par:

$$
\mathfrak{I}=\vartheta \mathrm{v}_{\mathrm{l}} / \vartheta \mathrm{x}_{\mathrm{k}}
$$

$v_{i}$ étant la vitesse de déplacement.

La déformation finie à un instant $t$ quelconque et notamment à l'état actuel s'obtient par intégration des équations du champ de vitesse de déformation (RAMBERG, 1975).

Exemple: déformation progressive bidimensionnelle isoaire (fig. 5b).

La vitesse de transformation est définie dans le référentiel constitué par les bissectrices des directions invariantes, par la matrice (I) :

$$
(\mathfrak{I})=\left(\begin{array}{cc}
0 & \dot{a}_{12} \\
\dot{a}_{21} & 0
\end{array}\right)
$$

Les déformations peu rotationnelles, à invariantes réelles d'angle $2 \mathrm{~V}$ sont caractérisées, à l'instant $\mathrm{t}$ par:

$$
(\mathrm{T})=\mathrm{Ch}(\mu \mathrm{t}) \cdot(\mathrm{I})+\operatorname{Sh}(\dot{\mu} \mathrm{t}) \frac{(\mathfrak{I})}{\dot{\mu}}
$$

(I) est la matrice identité et $\dot{\mu}^{2}=\dot{a}_{12}, \dot{a}_{21}>0$ avec comme cas particulier, la déformation pure $\mathrm{D}$, irrotationnelle, lorsque $\dot{a}_{12}=\dot{a}_{21}$ et $\mathrm{V}=\pi / 4$.

Les déformations très rotationnelles, sans directions invariantes, sont caractérisées, à l'instant t, par :

$$
(\mathrm{T})=\cos (\dot{\beta} \mathrm{t}) \cdot(\mathrm{I})+\sin (\dot{\beta} \mathrm{t}) \frac{(\mathfrak{I})}{\beta}
$$

où $\quad-\hat{\beta}^{2}=\dot{a}_{12} \dot{a}_{21}<0$

avec comme cas particulier, la rotation pure $\mathrm{R}$ (sans déformation), lorsque $\dot{a}_{12}=-\dot{a}_{21}=-\dot{\beta}$. 
initial
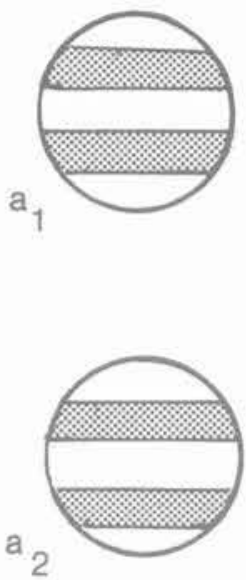

$a_{3}$

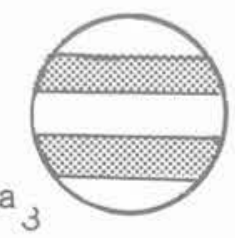

$a_{4}$

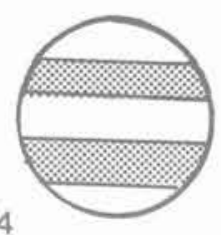

fin a I
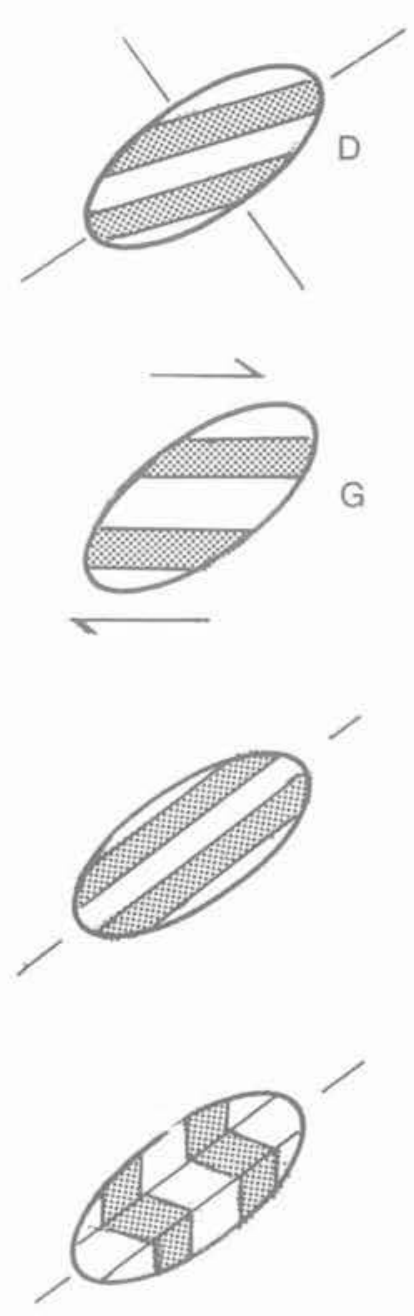

$b_{1}$

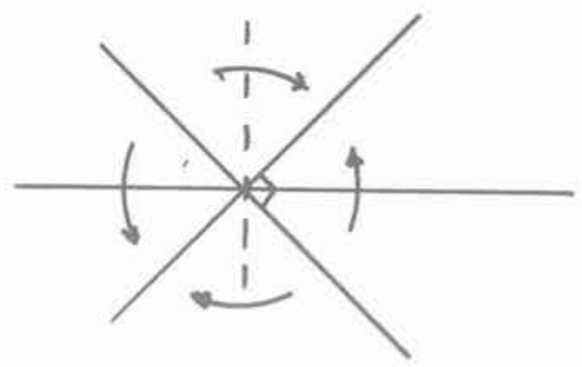

D

$b_{2}$
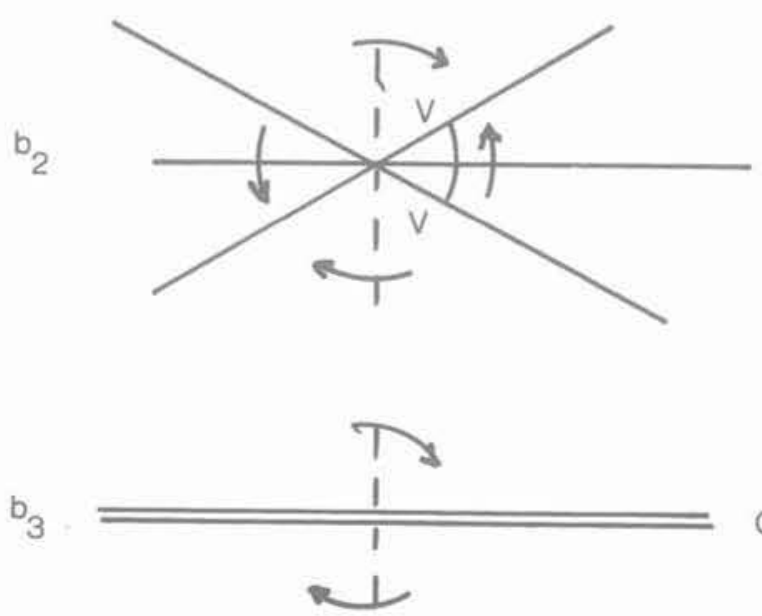

$\mathrm{G}$

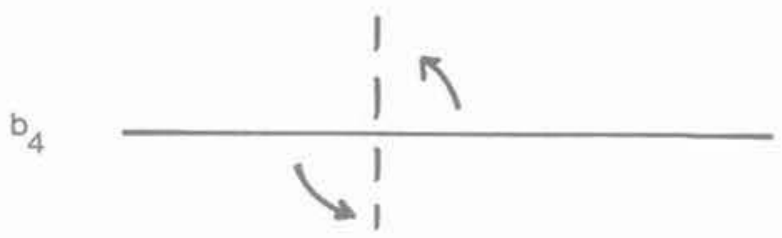

$b_{5}$

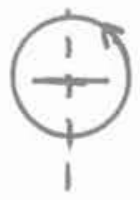

nal abc pour une structure à symétrie orthorhombique (planaire ou linéaire), un axe de révolution c (normal à un plan isotrope ab) pour une structure de révolution. L'exemple type est une schistosité $\mathrm{S}$ avec linéation $\mathrm{L}$ dans $\mathrm{S}$ : les axes géométriques abc sont tels que c est normal à $\mathrm{S}, \mathrm{b}$ confondu avec $\mathrm{L}$ dans $\mathrm{S}$, a orthogonal à b et c (fig. 6).

Les axes cinématiques sont définis par les trièdres principaux $\mathrm{X}_{\mathrm{d}} \mathrm{Y}_{\mathrm{d}} \mathrm{Z}_{\mathrm{d}}$ de (D) et $(\mathrm{XYZ})$ de $(\bar{D})$.

Dans le cas simple d'une déformation irrotationnelle, ces trois trièdres coïncident : la schistosité caractérise
Les axes géométriques caractérisent les éléments de symétrie d'une structure, ainsi un trièdre triorthogo-

\subsection{Structure et déformation}

La déformation finie et son chemin sont déterminés à l'aide des structures actuelles.

a. Chemins de déformation $\left(a_{1}=\right.$ déformation pure $b_{4}$ et $b_{5}=$ pas d'invariantes)

a. Strain path $\left(a_{7}=\right.$ pure shear, $a_{2}=$ sample shear,

Le cas intermédiaire caractérisé par une invariante

$$
(\mathrm{T})=(\mathrm{I})+(\mathfrak{I}) \cdot \mathrm{t}
$$

lorsque $\dot{a}_{21}=0$. 

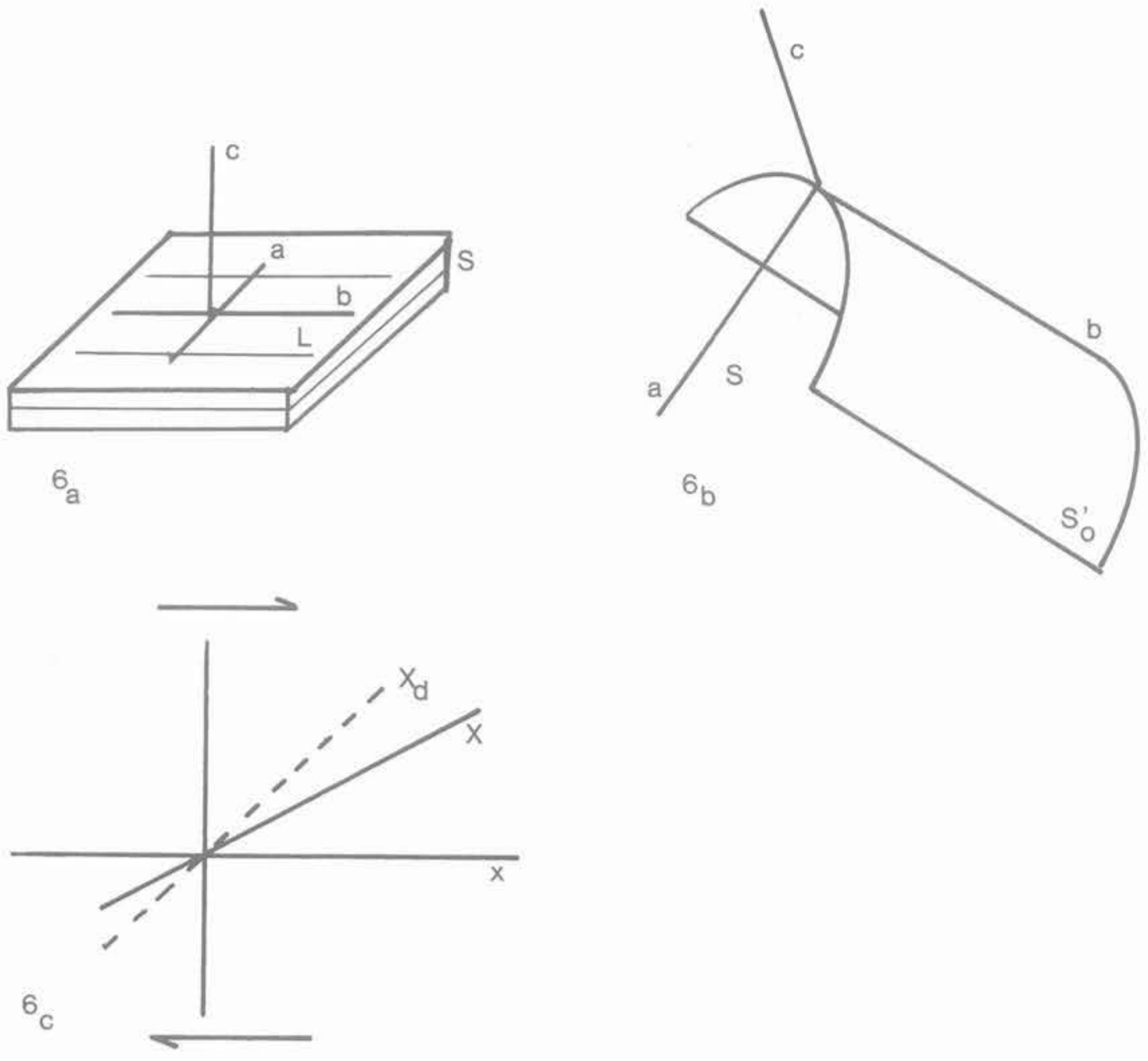

Fig. 6. - Axes géométriques et cinématiques.

$6 a: a b$ : plan de schistosité, $b$ : linéation; $6 b: a b=$ schistosité de plan axial de plis ; $6 c: X=$ trace de la schistosité.

Fig. 6 : - Geometrical and kinematic axis.

$6 a: a b$ : cleavage plane; $b$ : lineation; $6 b ; a b$ : axial plane cleavage; $6 c: X=$ cleavage intersection.

le plan $\mathrm{XY}$, la linéation marque la direction $\mathrm{X}$, avec la correspondance :

\section{XYZ}

bac

Dans la transformation rotationnelle de type glissement simple $\mathrm{G}$ (§ précédent), les axes principaux de $D$ sont les bissectrices de xy, la trace de la schistosité (ab) coincide avec la direction principale majeure $X$ de $(\bar{D}$ ), elle se réoriente donc constamment au cours de D (pour coïncider avec $\mathrm{X}$ ).

\section{SÉQUENCES DE LA DÉFORMATION}

\subsection{Phase et séquences de la déformation}

Un champ de vitesse de déformation, pour lequel l'orientation du trièdre principal de $D$ est constante, caractérise une phase de déformation tectonique. Il peut être arbitrairement décomposé en une superpo- sition dite simultanée, de champs de vitesse de déformation plus simples, par exemple vitesse de déformation pure et de glissement simple (RAMBERG, 1975).

La déformation tectonique observable est décomposée en phases successives, par superposition de déformations monophasées. Cette superposition, dite séquentielle, caractérise l'histoire c'est-à-dire la chronologie de la déformation. Chaque phase, définie en orientation et en intensité, se superpose à la précédente (exemple : déformation alpine superposée à la pyrénéo-provençale, GOGUEL, 1975). La déformation finale, actuelle, est le produit, non-commutatif en général, des déformations successives:

$$
\mathrm{T}=\mathrm{T}_{\mathrm{n}} \ldots \mathrm{T}_{3} \mathrm{~T}_{2} \mathrm{~T}_{1}
$$

(notation matricielle, lecture du produit de droite à gauche). Exemple; chemins biphasés de la figure $5 \mathrm{a}$. 
Les structures actuelles dépendent essentiellement de ces étapes (RAMBERG et al., 1976). La tectonométrie (quantification de la déformation tectonique et analyse de son chemin, généralement polyphasé) s'effectue à l'aide de marqueurs (fig. 7), tels que objets, fossiles, rejets, tectoglyphes (MATTAUER, 1980 ; FOUCAULT et RAOULT, 1984). L'hétérogénéité du milieu apporte de précieuses informations, par exemple éléments fracturés en mode I (boudins), fentes en échelon.

\subsection{Bifurcation - réactivation}

La déformation crée donc de nouvelles structures, notamment des structures de glissement. Par exemple, dans le cas d'un glissement simple: apparition de lignes (traces de plans) de glissement, appelées lignes de Riedel (P. VIALON et al., 1976) de première famille $R_{s}$ (synthétique) et de deuxième famille $R_{a}$ (antithétique), qui font l'angle $\pm \mu$ avec la direction de contraction majeure $Z_{\mathrm{d}}$ (fig. 8).

$\mathrm{Au}$ cours de son histoire, la déformation peut changer de régime, bifurquer, en utilisant les nouvelles

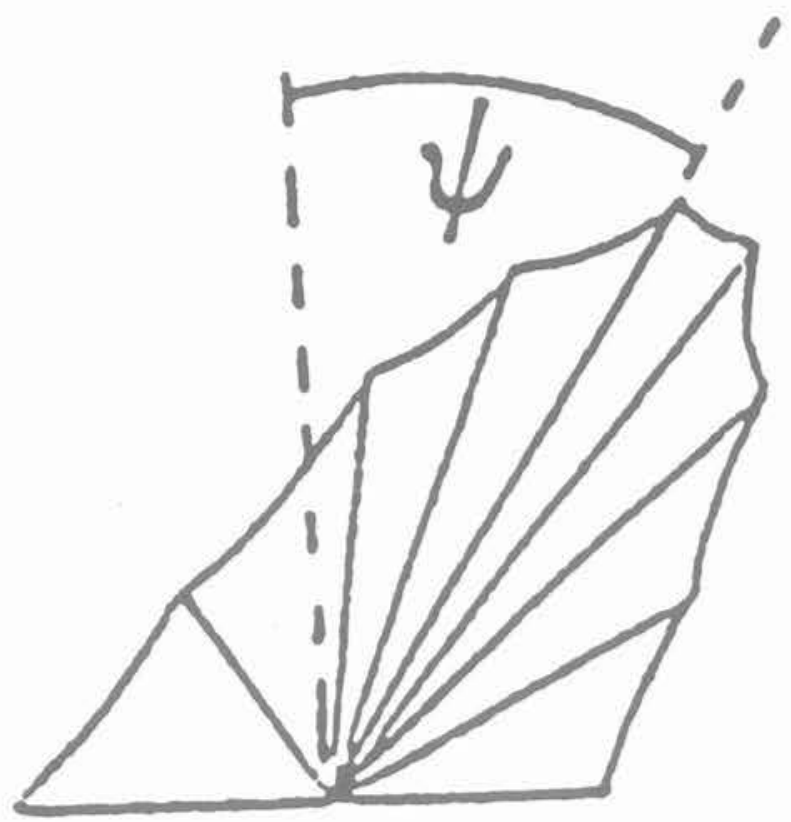

a

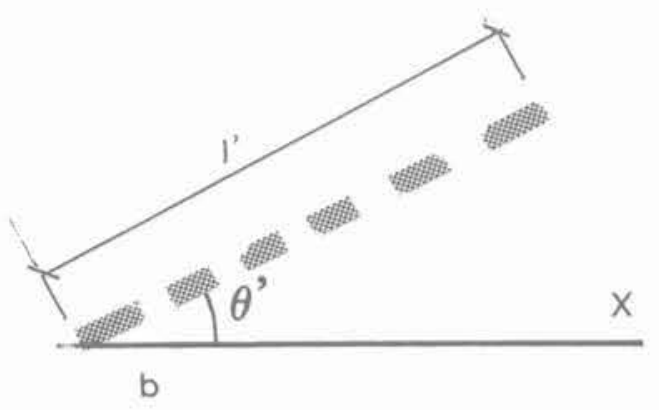

Fig. 7. - Marqueurs. a. Distorsion (fossile déformé) ; b. Extension (boudins).

Fig. 7. - Markers. a. Distorsion (strained fossill): b. Extension (boudins).

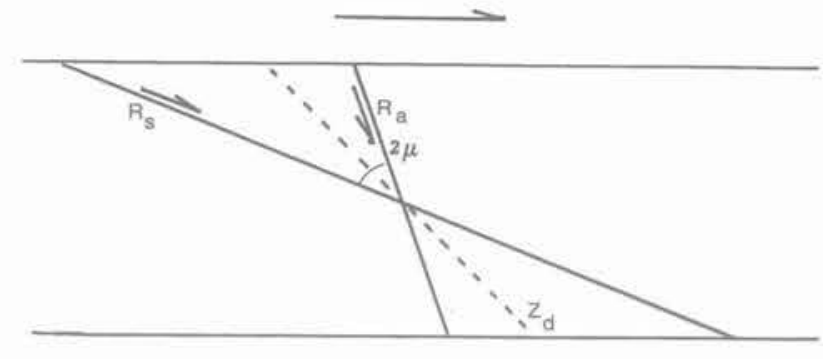

Fig. 8. - Lignes de Riedel. $R_{s}$ (synthétique), $R_{a}$ (antithétique).

Fig. 8. - Riedel lines. $R_{S}$ (synthetic), $R_{a}$ (antithetic).

structures créées. Dans le cas précédent du glissement simple, le glissement peut se poursuivre par bifurcation, sur $R_{s}$ (Riedel synthétique) et ainsi de suite, avec apparition de fissures branchées.

De même au cours d'une phase ultérieure, la déformation peut utiliser des structures préexistantes, du fait de l'anisotropie du milieu (cas de réactivation de failles ou de structures antérieures) ou au contraire s'effectuer sans faire intervenir c'est-à-dire sans réactiver de surface structurale antérieure naturelle, auquel cas la structure est dite passive. Exemple de réactivation: dans un système de deux familles de failles conjuguées, une nouvelle phase de déformation s'effectuera par glissement sur l'une des familles de failles selon son orientation et ses capacités de réactivation, c'est-à-dire selon les valeurs des constantes rhéologiques structurales.

\section{CHAMPS DE CONTRAINTES}

Les phases de déformation étant déterminées, les champs cinématiques étant définis par les enveloppes des directions principales, les champs de contraintes sont également définis par leur trièdre principal $\mathrm{X}_{\sigma} \mathrm{Y}_{\sigma} \mathrm{Z}_{\sigma}$ et les isostatiques. Leur orientation par rapport aux trièdres cinématiques, de même que leur intensité, nécessite la connaissance de données rhéologiques. Il est possible de déterminer dans certains cas lorientation des paléocontraintes. L'état de contraintes actuel est sans relation avec la déformation totale, il est relié au champ de vitesse de déformation actuelle, qui fait l'objet des études néotectoniques.

Dans un système de failles conjuguées les directions principales des contraintes génétiques $\mathrm{X}_{\sigma} \mathrm{Y}_{\sigma} \mathrm{Z}_{\sigma}$ sont telles que $\mathrm{Y}_{\sigma}$ est confondu avec le rectiligne du dièdre, $Z_{\sigma}$, caractérisant la compression majeure, coïncide avec la bissectrice de l'angle aigu des traces des deux failles (fig. 9a).

Lors de la réactivation d'une faille, selon le mécanisme du glissement structural, il est admis (BOTT, 1959) que dans le plan de faille la direction de la contrainte de cisaillement est coaxiale à celle de la strie (fig. 9b). Dans le cas général, un état de contrainte appliqué à une structure planaire $\mathrm{S}$ fait intervenir des plages d'orientations structurales. Par exemple, lorsque $\mathrm{Y}_{o}$ est situé dans $\mathrm{S}$, selon l'orientation de $Z_{a}$ il est possible d'aboutir à une structure plissée (kink-bands notamment), à un glissement structural sur $\mathrm{S}$, à une déformation homogène avec possibilité de création d'une nouvelle structure (fig. 9c). 

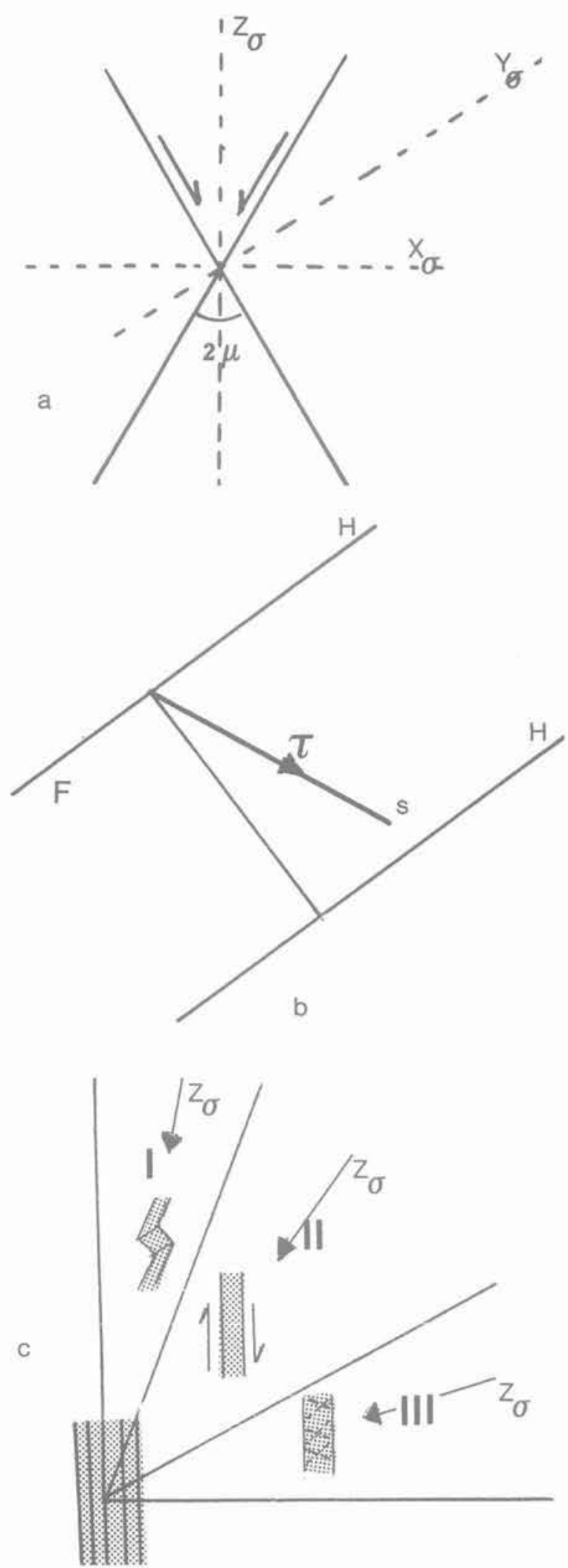

Fig. 9. - Trièdre des contraintes.

9a: Failles conjuguées; $9 b$ : Glissement structural : $\tau=$ composante de cisaillement $s=$ strie)

9c. : Anisotropie discontinue : mécanismes I (plissement), II (glissement structural) et III (néostructure).

Fig. 9. - Stress axis, a : Conjugate faults ; $b$ : Structural slip $\tau=$ shear component, $s=$ striae) $; c$ : discontinuous anisotropy mechanism I (folding), II (structural slip) and III (neostructur).
Finalement, l'étude de l'histoire d'un massif consiste à analyser les orientations relatives des quatre trièdres: $a b c$ (de la structure), XYZC de $\bar{D}$ ), $X_{d} Y_{d} Z_{d}$ (de D) et $\mathrm{X}_{\sigma} \mathrm{Y}_{\sigma} \mathrm{Z}_{\sigma}$ (de $\left.\Sigma\right)$.

\section{CONCLUSION}

Les observations et mesures de terrain permettent, dans certains cas, de retracer l'histoire cinématique des massifs. Les résultats de la tectonométrie fournissent les champs de déformation finie, de vitesse de déformation donc des différentes phases et éventuellement les champs de contraintes; ils apportent des informations sur le comportement des roches concernées (par exemple : compétence relative des bancs dans le plissement, intensité de la déformation selon l'épaisseur des bancs). Ils permettent en outre d'envisager les déformations susceptibles de se produire lors de nouvelles sollicitations.

\section{BIBLIOGRAPHIE}

BOTT M.H.D. (1959), The mechanics of oblique slip faulting. Geol. Mag., 96, p. 107-109.

FOUCAULT A., RAOULT J.F. (1984), (2e édition), Dictionnaire de Géologie. Masson, Paris, 347 p.

GOGUEL J. (1975), (6 édition), (1 re édition en 1950), Géologie de la France. Que sais-je? PUF, $128 \mathrm{p}$

GOGUEL J. (1979), Analyse théorique du chemin de la déformation, à gradient de vélocité permanent. Tectonophysics. 60, T 17-T 26.

MATTAUER M. (1980), Les déformations des matériaux de l'écorce terrestre. Paris, Hermann, 4e édition, $433 \mathrm{p}$.

RAMBERG H. (1975), Superposition of homogeneous strain and progressive deformation in rocks. Bull. Geol. Inst. Univ. Uppsala. NS Vol. 6, p. 35-67.

RAMBERG H., GHOSH S.K. (1976), Reorientation of inclusions by combination of pure shear and simple shear. Tectonophysics, $n^{\circ} 34, p, 1-70$.

RAMSAY J. (1967), Folding and fracturing of rocks. Mc Graw Hill. 568 p.

SIRIEYS P. (1984), Déformation homogénéisée des roches par glissements hétérogènes continus et discontinus. Bull. Soc. Geol. Fr. (7), t. XXVI, $\mathrm{n}^{\circ} 1, \mathrm{p} ; 185-192$.

VIALON P., RUHLAND M., GROLIER J. (1976), Eléments de tectonique analytique. Masson, Paris, $118 \mathrm{p}$. 